\title{
Molecular genetics and clinical features of Chinese idiopathic and heritable pulmonary arterial hypertension patients
}

\author{
D. Liü ${ }^{\star,}$, Q-Q. Liu ${ }^{\#,+}$, M. Eyries ${ }^{\star}$, W-H. Wu*, P. Yuan*, R. Zhang*, \\ F. Soubrier and Z-C. Jing*
}

ABSTRACT: Mutations of the bone morphogenetic protein type II receptor (BMPR2) gene predispose to pulmonary arterial hypertension (PAH). 290 idiopathic (I)PAH patients and 15 heritable (H)PAH were screened to determine the spectrum and rate of BMPR2 mutations in a large Chinese patient group.

Gene sequencing and multiplex ligation-dependent probe amplification (MLPA ${ }_{R}$ ) were used to detect sequence mutations and large rearrangements (RGTs). Total mutation rate was $14.5 \%$ ( $n=42$ out of 290$)$ in Chinese IPAH patients, and $53.3 \%(n=8$ out of 15$)$ in HPAH patients. RGT mutation rate was $3.1 \%$ ( $n=7$ out of 229$)$ and represented $14 \%(n=7$ out of 50$)$ of all identified mutations. 25 BMPR2 mutations were newly identified.

Patients in this study were younger than other reported PAH subjects. BMPR2 mutation carriers were $\sim 6$ yrs younger at diagnosis than noncarriers $(p=0.002)$, but this relationship was significant only in the female group, which was larger. The proportion of females carrying a BMPR2 mutation was half that of males $(12.8 \%$ versus $25.3 \% ; p=0.008)$.

Our results indicate that the overall genetics of Chinese PAH patients is similar to that of other populations, but the clinical picture differs by the precocity of the disease in the whole patient group, and the lower proportion of females found to carry a BMPR2 mutation.

KEYWORDS: BMPR2 mutation, genetics, pulmonary circulation

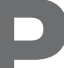
ulmonary arterial hypertension (PAH) is a rare and potentially fatal disorder characterised by plexiform lesions of proliferating endothelial cells and smooth muscle cells in pulmonary arterioles, which lead to elevated pulmonary artery pressures, right ventricular failure and death [1]. At least $6 \%$ of cases have a recognised family history, in which the disease segregates as an autosomal dominant trait with incomplete penetrance and an estimated lifetime risk of 10$20 \%$ [2]. The disease is more frequent in females, with a female/male ratio of at least 1.7:1 [1, 3, 4]. The average age of onset is in the third decade of life, but there is wide variation within families, and childhood onset is common.

Familial cases have long been recognised and mutations in the bone morphogenetic protein type II receptor (BMPR2) gene (BMPR2) have been recognised to cause heritable $(\mathrm{H}) \mathrm{PAH}[5,6]$. Subsequent studies have reported $>250$ BMPR2 mutations responsible for $55-70 \%$ of heritable
(H)PAH, and $11-40 \%$ of sporadic idiopathic (I)PAH cases [7, 8]. After the last world conference on pulmonary hypertension, these forms were grouped under the term heritable [9].

BMPR2 encodes the bone morphogenetic protein type II receptor, a member of the transforming growth factor (TGF)- $\beta$ cell signalling superfamily [5-7]. After ligand binding, type II receptors, which have serine/threonine kinase activity, form heteromeric complexes with membrane-bound type I receptors, initiating phosphorylation of the type I receptor and downstream intracellular Smads or mitogen-activated protein kinases (MAPKs) [10, 11]. This pathway appears to be critical in both cell differentiation and growth, through transcriptional regulation of target genes.

To improve knowledge of the genetic origin of $\mathrm{PAH}$ in Chinese patients with the disorder, we investigated 15 unrelated families with $\mathrm{PAH}$ and 290 sporadic $\mathrm{PAH}$ patients for BMPR2 mutations.
AFFILIATIONS

*Dept of Cardio-Pulmonary Circulation, Shanghai Pulmonary Hospital, Tongji University, School of Medicine, Shanghai, and \#Dept of Cardiology, the 4th Affiliated Hospital, Harbin Medical University, Harbin, China. - Université Pierre et Marie CurieParis 6, Laboratoire d'Oncogénétique et Angiogénétique Moléculaire, Unité Mixte de Recherche en Santé 956, Institut National de la Santé et de la Recherche Médicale, Groupe Hospitalier Pitié-Salpêtrière, Paris, France.

${ }^{+}$These authors contributed equally to this work.

CORRESPONDENCE

Z-C. Jing

Dept of Cardio-Pulmonary Circulation Shanghai Pulmonary Hospital Tongji University

School of Medicine

No. 507 Zhengmin Road Shanghai 200433

China

E-mail: jingzhicheng@gmail.com

Received:

May 042011

Accepted after revision: June 262011

First published online: July 072011 
Direct sequencing to detect mutations in coding regions and flanking splice sites, in addition to analysis of exon dosage across the entire gene using multiplex ligation-dependent probe amplification (MLPA ${ }_{\mathbb{R}}$ ) showed a wide BMPR2 mutation spectrum in Chinese patients with PAH. Our study provides an extensive investigation of the genetic aetiology of Chinese heritable and idiopathic $\mathrm{PAH}$, which gives insight into the variety of BMPR2 mutations among different ethnic groups.

\section{METHODS}

\section{Study patients}

All patients belonged to the Chinese Han population and visited Shanghai Pulmonary Hospital, Shanghai, China, between January 1, 2006 and August 31, 2010. 290 IPAH and 15 HPAH patients were tested for BMPR2 point mutations and large size rearrangements. The diagnosis of IPAH required the presence of elevated mean pulmonary artery pressure $\left(\bar{P}_{\text {pa }}>25 \mathrm{mmHg}\right.$, with a pulmonary capillary wedge pressure $\leqslant 15 \mathrm{mmHg}$ measured by right heart catheterisation at rest), and the exclusion of other disorders known to cause pulmonary hypertension by clinical evaluation and objective tests. HPAH was recognised if there was more than one confirmed case in first- to third-degree relatives in the family [12]. A single proband from each affected pedigree was included in the study $(n=15)$. All participants gave their written informed consent for genetic analyses prior to participation. The study was approved by the Ethics Committee of Shanghai Pulmonary Hospital.

\section{Molecular methods}

Direct screening using an ABI 3730 DNA analyser (Applied Biosystems, Carlsbad, CA, USA) was adopted to detect point mutations in the coding regions and intron/exon boundaries of BMPR2. Genomic DNA was isolated from peripheral blood leukocytes. 15 pairs of PCR primers were designed to amplify

\begin{tabular}{|c|c|c|c|c|}
\hline \multirow[t]{2}{*}{ TABLE 1} & \multicolumn{4}{|c|}{$\begin{array}{l}\text { Clinical characteristics of the study participants } \\
\text { at the time of diagnosis }\end{array}$} \\
\hline & All & HPAH & IPAH & p-value ${ }^{\#}$ \\
\hline Subjects $\mathrm{n}$ & 305 & 15 & 290 & \\
\hline Age yrs & $33 \pm 16$ & $20 \pm 10$ & $34 \pm 16$ & $<0.001$ \\
\hline Sex male/female $n$ & $87 / 218$ & $6 / 9$ & $81 / 209$ & $0.379^{\circ}$ \\
\hline Height $\mathrm{cm}$ & $158 \pm 17$ & $153 \pm 25$ & $158 \pm 17$ & 0.215 \\
\hline Weight kg & $54 \pm 16$ & $48 \pm 20$ & $55 \pm 16$ & 0.125 \\
\hline 6MWD m & $381 \pm 113$ & $386 \pm 119$ & $381 \pm 113$ & 0.893 \\
\hline $\bar{P}_{\text {pa }} \mathrm{mmHg}$ & $62 \pm 17$ & $71 \pm 15$ & $62 \pm 17$ & 0.053 \\
\hline $\bar{P}_{\text {pcw }} \mathrm{mmHg}$ & $8 \pm 5$ & $9 \pm 4$ & $8 \pm 5$ & 0.870 \\
\hline PVR Wood units & $17 \pm 10$ & $23 \pm 12$ & $17 \pm 10$ & 0.042 \\
\hline CO L.min ${ }^{-1}$ & $3.8 \pm 1.5$ & $3.2 \pm 1.4$ & $3.8 \pm 1.5$ & 0.271 \\
\hline $\mathrm{CI} \mathrm{L} \cdot \mathrm{min}^{-1} \cdot \mathrm{m}^{-2}$ & $2.5 \pm 1.0$ & $2.6 \pm 1.5$ & $2.5 \pm 1.0$ & 0.598 \\
\hline
\end{tabular}

Data are presented as mean $\pm \mathrm{SD}$, unless otherwise stated. \#: p-values represent the results of independent unpaired t-tests comparing heritable $(\mathrm{H})$ and idiopathic (I) pulmonary arterial hypertension (PAH) patients, with $p<0.05$ chosen as the level of significance; ": Fisher's exact test for the comparison of sex for HPAH and IPAH. 6MWD: 6-min walk distance; $\bar{P}$ pa: mean pulmonary arterial pressure; $\bar{P}_{\mathrm{pcw}}$ : mean pulmonary capillary wedge pressure; PVR: pulmonary vascular resistance; $\mathrm{CO}$ : cardiac output; $\mathrm{Cl}$ : cardiac index.
13 exons and the $5^{\prime}$ - and $3^{\prime}$-untranslated regions of the BMPR2 gene (see online supplementary table 1 ). The results were compared with the reference sequence of the BMPR2 gene (RefSeq accession number NM_001204.5) with the ABI SeqScape software, version 2.5 (Applied Biosystems). The mutation nomenclature followed current guidelines as recommended by the Human Genome Variation Society [13]. The mutation numbering employed in this report is based on the cDNA sequence, where +1 designates the A of the ATG initiation codon.

The BMPR2 gene was screened for large size rearrangements (RGTs) using the SALSA MLPA ${ }_{\circledast}$ P093 HHT probe mix kit (MRC-Holland BV, Amsterdam, The Netherlands), according to the manufacturer's instructions. Samples were analysed on an ABI 377 fluorescent analyser with GeneScan and GenoTyper software (Applied Biosystems, Warrington, UK; www.appliedbiosystems.com). RGTs were analysed by the Coffalyser software (MRC-Holland BV). Two DNA samples from unaffected individuals were used as controls in each series of experiments. Electrophoregrams were superimposed on those generated with a control DNA by adjusting to the same levels the peaks obtained for the control amplicons. Only PAH patients for whom no BMPR2 mutation could be detected by direct sequencing were analysed for RGTs $(n=255) .232$ results (229 IPAH and three HPAH) were readable because of technical problems on the remaining samples.

The coding sequences and exon-intron boundaries of the ACVRL1 and ENG genes in seven HPAH patients negative for $B M P R 2$ mutations were also sequenced.

\section{Statistical analysis and bioinformatic tools}

We compared the demographic and basic clinical features of IPAH and HPAH patients with the use of Chi-squared, Fisher's exact t-tests, where appropriate. A p-value of $<0.05$ was considered to indicate statistical significance. Polyphen-2 software (http:/ /genetics.bwh.harvard.edu/pph2) was used for in silico analysis of coding variants, and splice site variants were analysed by Splice Site Finder, MaxEntScan, Nnsplice and Genesplicer, all available with Alamut v1.5 software (Interactive Biosoftware, Rouen, France).

\section{RESULTS \\ Clinical characteristics of the study participants}

The study population included 290 patients with sporadic idiopathic PAH and 15 probands from unrelated pedigrees with heritable PAH (15 families; table 1$)$; 87 were male. The mean \pm SD age of the patients was $33 \pm 16$ (range 1-77) yrs. $\mathrm{HPAH}$ patients were $\sim 14$ yrs younger than those with IPAH $(20 \pm 10$ versus $34 \pm 16$ yrs; $\mathrm{p}<0.001)$.

\section{Mutation rate and distribution}

Mutation analysis in HPAH and IPAH is summarised in tables 2 and 3. A total of 50 mutations were identified, accounting for eight out of the 15 patients with HPAH (53.3\%) and 42 out of the 290 patients with IPAH (14.5\%). 25 mutations are reported for the first time.

49 out of the 50 patients had nonsynonymous BMPR2 variations (table 2). Classification of these variants is shown in table 4.

Four variants were found in at least two unrelated patients. Exon 10 deletion of BMPR2 was found in two IPAH patients and 
TABLE 2 BMPR2 mutations in Chinese patients with idiopathic (I) and heritable $(\mathrm{H})$ pulmonary arterial hypertension (PAH)

\begin{tabular}{|c|c|c|c|c|c|c|c|}
\hline $\begin{array}{l}\text { Identification } \\
\text { number }\end{array}$ & $\begin{array}{l}\text { IPAH or } \\
\text { HPAH }\end{array}$ & Location & Domain & $\begin{array}{l}\text { Nucleotide } \\
\text { change }^{\#}\end{array}$ & $\begin{array}{l}\text { Amino acid } \\
\text { change }{ }^{\pi}\end{array}$ & $\begin{array}{c}\text { Mutation } \\
\text { type }\end{array}$ & [Ref.] \\
\hline 63 & IPAH & $5^{\prime}$-UTR & & c. $-310 A>G$ & p.? & VUS & This study \\
\hline 403 & IPAH & exon1 & & c.21delG & p.Trp9GlyfsX38 & Frameshift & This study \\
\hline 375 & IPAH & exon1 & & c. $27 \mathrm{G}>\mathrm{A}$ & p.Trp9x & Nonsense & [14] \\
\hline 307 & $\mathrm{IPAH}$ & exon2 & ECD & c. $88 \bar{C}>T$ & p.Gln30X & Nonsense & This study \\
\hline 336 & $\mathrm{IPAH}$ & exon2 & ECD & c. 103delG & p.Ala35ArgfsX12 & Frameshift & This study \\
\hline 1143 & IPAH & exon2 & ECD & c. $178 \mathrm{~T}>\mathrm{G}^{+}$ & p.Cys60Gly & Missense & This study \\
\hline 1016 & IPAH & exon3 & ECD & c. $251 \mathrm{G}>\mathrm{T}^{+}$ & p.Cys84Phe & Missense & This study \\
\hline 1063 & HPAH & exon3 & ECD & c. $266 G>C$ & p.Gly89Ala & Missense & This study \\
\hline 1001 & IPAH & exon3 & ECD & c. $338 \mathrm{~A}>\mathrm{G}^{+}$ & p.Tyr 113 Cys & Missense & This study \\
\hline 467 & HPAH & exon4 & ECD & c. $439 C>T$ & p.Arg147X & Nonsense & {$[8]$} \\
\hline 468 & HPAH & exon4 & ECD & c. $439 \mathrm{C}>\mathrm{T}$ & p.Arg147X & Nonsense & [8] \\
\hline 913 & $\mathrm{IPAH}$ & exon6 & $K D$ & c. $830 \mathrm{~T}>\mathrm{C}$ & p.Leu227Pro & Missense & [15] \\
\hline 498 & IPAH & intron7 & $\mathrm{KD}$ & c. $967+5 G>C$ & p.? & VUS & This study \\
\hline 370 & IPAH & exon8 & $\mathrm{KD}$ & c. $969 \mathrm{~T}>\mathrm{C}$ & p.Asp323Asp & Synonymous & This study \\
\hline 209 & IPAH & exon8 & $\mathrm{KD}$ & c. $994 \mathrm{C}>\mathrm{T}$ & p.Arg332X & Nonsense & {$[7,14,17]$} \\
\hline 28 & IPAH & exon8 & $\mathrm{KD}$ & c. $1042 G>A$ & p.Val348lle & Missense & {$[16,18]$} \\
\hline 94 & IPAH & exon8 & $\mathrm{KD}$ & c. $1042 G>A$ & p.Val348lle & Missense & {$[16,18]$} \\
\hline 711 & IPAH & exon8 & $K D$ & c.1093_1098delinsG & p.Arg365GlyfsX5 & Frameshift & This study \\
\hline 534 & IPAH & exon8 & $\mathrm{KD}$ & c. $1117 G>C$ & p.Ala373Pro & Missense & This study \\
\hline 326 & IPAH & intron8 & $\mathrm{KD}$ & C. $1129-3 C>G$ & p.? & Splice defect & {$[14,17]$} \\
\hline 141 & IPAH & exon9 & $\mathrm{KD}$ & C. $1175 T>C$ & p.Val392Ala & Missense & [18] \\
\hline 962 & HPAH & exon11 & $K D$ & c. $1471 C>T$ & p.Arg491Trp & Missense & [5] \\
\hline 869 & IPAH & exon11 & $\mathrm{KD}$ & c. $1472 G>A$ & p.Arg491Gln & Missense & [5] \\
\hline 1382 & IPAH & exon11 & $K D$ & c. $1472 G>A$ & p.Arg491Gln & Missense & [5] \\
\hline 379 & IPAH & exon12 & $\mathrm{CD}$ & c. $2296 A>G$ & p.Thr766Ala & Missense & This study \\
\hline 853 & IPAH & exon12 & $C D$ & c.2446_2447dup & p.Asn817Leufsx23 & Frameshift & This study \\
\hline 52 & IPAH & exon12 & $C D$ & c.2503_2506del & p.Thr835ProfsX3 & Frameshift & This study \\
\hline 499 & IPAH & exon12 & $C D$ & c. $2617 \mathrm{C}>\mathrm{T}$ & p.Arg873X & Nonsense & {$[5,14]$} \\
\hline 432 & IPAH & exon12 & $\mathrm{CD}$ & c. $2695 \mathrm{C}>\mathrm{T}$ & p.Arg899X & Nonsense & {$[6,7,8,14,17]$} \\
\hline 81 & HPAH & exon13 & $C D$ & c. $3117+12 G>A$ & p.? & VUS & This study \\
\hline
\end{tabular}

\#: abbreviations are in accord with nomenclature guidelines as recommended by the Human Genome Variation Society [13]; the letter c. indicates coding DNA, where nucleotide 1 is the A of the ATG translation initiation codon; " : the letter $p$. is used to indicate the change at the protein level; ${ }^{+}$: missense mutation predicted to be probably damaging by Polyphen-2 software. UTR: untranslated region; ECD: extracellular domain; KD: kinase domain; CD: cytoplasmic domain; VUS: variant of unknown significance.

has already been reported, but the breakpoints were not determined. The remaining 37 patients had unique BMPR2 mutations.

These mutations are distributed from the $5^{\prime}$-UTR to exon 13 of $B M P R 2$. No mutations in exon 5 or exon 7 were found either in the IPAH or the HPAH cohort.

\section{Point mutations}

15 out of the 43 point mutations were either frameshift $(n=6)$ or nonsense $(n=9)$ mutations that were predicted to result in a truncated BMPR2 molecule. All six frameshift mutations were newly identified insertions or deletions. Among the 21 missense mutations, eight were novel mutation sites. The effect on protein function was predicted with PolyPhen-2 [21]. 
TABLE 3 Pulmonary arterial hypertension (PAH) patients identified with BMPR2 rearrangements

\begin{tabular}{lccc} 
Identification number & Diagnosis & Rearrangement & [Ref.] \\
\hline $\mathbf{1 2 8 4}$ & IPAH & Dup BMPR2 exon 2-3 c.77-?_c.421+?dup & This study \\
$\mathbf{7 4}$ & IPAH & Dup BMPR2 exon 2-7 c.77-?_c.967+?dup & This study \\
$\mathbf{3 6 1}$ & IPAH & Dup BMPR2 exon 8-10 c.968-?_c.1413+?dup & This study \\
$\mathbf{2 9 4}$ & IPAH & Dup BMPR2 exon 10 c.1277-?_c.1413+?dup & This study \\
$\mathbf{8 6 0}$ & IPAH & Del BMPR2 exon 10 c.1277-?_c.1413+?del & [19] \\
$\mathbf{1 2 4 7}$ & IPAH & Del BMPR2 exon 10 c.1277-?_c.1413+?del & [19] \\
$\mathbf{8 9 5}$ & IPAH & Del BMPR2 exon 11-13 c.1414-?_c.3117+?del & [20] \\
\hline
\end{tabular}

\#: abbreviations are in accord with nomenclature guidelines as recommended by the Human Genome Variation Society [13]. IPAH: idiopathic PAH; Del/del: deletion Dup/dup: duplication; c.: coding DNA where nucleotide 1 is the A of the ATG translation initiation codon.

Five mutations (table 2) were predicted to be probably damaging, while the remaining three mutations (c.266G $>C$, c. $1117 \mathrm{G}>\mathrm{C}$ and c.2296A $>\mathrm{G}$ ) were predicted to be possibly damaging. These three mutations were not found in 200 control chromosomes. Two splice-site mutations were found in IPAH patients. Variant c.1129-3C $>$ G has been reported before $[14,17]$, whereas the other was identified in this study. Variants of unknown significance found in the 5'-UTR (c.-223A $>$ G, c. $-310 A>G$ ), in intron 7 (c.967+5G>C), and in the $3^{\prime}$-UTR (c.3117+12G>A) are reported for the first time in this study. Variant c. $967+5 \mathrm{G}>\mathrm{C}$ and the synonymous mutation c.969T $>C$ that affected the second base of exon 8 were analysed by splice-site prediction programs. A possible effect on splicing was predicted for these two variants (see online supplementary table 2). However, further analyses on RNA are needed to draw a conclusion on the deleterious effect of these variants.

\section{Large rearrangements}

255 IPAH patients for whom no mutation was detected by sequencing were analysed by MLPA ${ }_{\circledR}$. Only 229 results were readable among the 255 IPAH patients, and seven RGTs were found. The RGT mutation rate is $3.1 \%$ in IPAH patients who had no sequencing mutations (seven out of 229 IPAH patients). Among $15 \mathrm{HPAH}$ patients, eight were found with mutation by sequencing and seven were analysed by MLPA®. Only three DNAs from seven HPAH patients could be analysed, and none was found with RGT mutation. Among these seven RGTs, four are newly identified (table 3).

Overall, RGTs represented $14 \%$ of all mutations in the Chinese PAH cohort.

\section{Polymorphisms}

Four nucleotide changes were considered to represent polymorphisms because they were also observed in control individuals (online supplementary table 3). Polymorphism c.1-93A $>\mathrm{G}$ was found in eight patients in the $5^{\prime}$-UTR and is first reported in this study. 237 normal Chinese Han people were screened and four patients were found to be carrying the same variant. The allele frequency is $0.84 \%$ for the $\mathrm{G}$ allele.

\section{ACVRL1 and ENG in HPAH}

The coding sequences and intron-exon boundaries of these two genes were analysed in index cases of PAH families for whom no mutation was found in the BMPR2 gene. A single missense mutation was found in the ACVRL1 gene, and is considered as potentially damaging according to PolyPhen-2 software.

\section{Age at diagnosis in mutation carriers and noncarriers}

The mean age at diagnosis was compared in carriers and noncarriers of mutations and was found to be significantly lower in carriers $(28 \pm 11 \mathrm{yrs}, \mathrm{n}=50)$ than in noncarriers $(34 \pm 17$ yrs, $n=255) \quad(p<0.002)$. The mean age at onset was found to be significantly lower in females carrying a BMPR2 mutation $(29 \pm 12$ yrs, $n=28)$ than in those without mutation $(35 \pm 16$ yrs, $n=190 ; p=0.036)$. In the male group, which was smaller, the age difference between male carriers $(27 \pm 10 \mathrm{yrs}$, $\mathrm{n}=22)$ and noncarriers $(32 \pm 19$ yrs, $n=65)$ did not reach statistical significance $(\mathrm{p}=0.071)$ (table 5$)$.

\section{DISCUSSION}

A total of 298 BMPR2 mutations have been identified until now in independent PAH patients [14, 16], including those with a

TABLE 4 Categories of BMPR2 mutations found in Chinese patients with idiopathic (I) and heritable $(H)$ PAH

\begin{tabular}{lcccccccc} 
Patient & Missense & Nonsense & Frameshift & Splice Site & Synonymous & VUS & RGT & Total \\
\hline IPAH & 16 & 7 & 6 & 2 & 1 & 3 & 7 \\
HPAH & 5 & 2 & 0 & 0 & 0 & 42 & 0 & 8 \\
Total & $21(42)$ & $9(18)$ & $6(12)$ & $2(4)$ & $1(2)$ & $4(8)$ & $7(14)$ & $50(100)$ \\
\hline
\end{tabular}

Data are presented as n or n (\%). VUS: variant of unknown significance; RGT: rearrangement. 


\begin{tabular}{|c|c|c|c|}
\hline TABLE 5 & $\begin{array}{l}\text { Mean age at puln } \\
\text { diagnosis in fema } \\
\text { BMPR2 mutation }\end{array}$ & $\begin{array}{l}\text { onary arterial hyper } \\
\text { es and males acco } \\
\text { tatus }\end{array}$ & $\begin{array}{l}\text { insion } \\
\text { ling to }\end{array}$ \\
\hline Age yrs & Mutation carrier & Nonmutation carrier & p-value ${ }^{\#}$ \\
\hline Female & $29 \pm 12(28)$ & $35 \pm 16(190)$ & 0.036 \\
\hline Male & $27 \pm 10(22)$ & $32 \pm 19(65)$ & 0.071 \\
\hline Total & $28 \pm 11(50)$ & $34 \pm 17(255)$ & 0.002 \\
\hline
\end{tabular}

known PAH family history or a sporadic onset of the disease. To date, mutations are usually found in around $70 \%$ of individuals with $\mathrm{HPAH}$, whereas the mutation detection rate ranges from $10 \%$ to $40 \%$ in $\mathrm{IPAH}[7,8]$. In this study, we found mutation rates in Chinese Han IPAH and HPAH patients of $14.5 \%$ and $53.3 \%$, respectively. These mutation detection rates are in a similar range to those reported in the literature and, in particular, by WANG et al. [18] who reported 12 mutations in 72 Chinese IPAH patients (16.7\%) without searching for RGT mutations by MLPA ${ }_{\circledR}$.

It is worth noting that the proportion of mutations found in groups of PAH patients is comparable in all published series, whatever the population of origin. Either these proportions are the consequences of technical limitations in detecting a particular type of mutations, or a relatively constant proportion of HPAH or IPAH among populations is secondary to other causes that remain to be elucidated.

Mutations predicted to introduce premature truncation codons to the BMPR2 open-reading frame in the present study encompass nonsense (nine out of 50;18\%), frameshift (six out of $50 ; 12 \%$ ), splice site (two out of $50 ; 4 \%$ ) and gene duplications / deletions (seven out of $50 ; 14 \%$ ). The total proportion of mutations found to cause premature termination was $48 \%$, much less than the $68 \%$ reported in 2009 by MACHADO et al. [16].

We used MLPA ${ }_{\circledR}$ to detect large gene RGTs which underlie a significant proportion of HPAH and IPAH cases [19, 22]. In our study, the proportion of RGTs was $14 \%$ of all the mutation categories, and they were present in 3.1\% (seven out of 229) of Chinese Han sporadic IPAH patients negative for BMPR2 mutation by DNA sequencing. These proportions are close to those observed by ALDRED et al. [19] who reported a proportion of $12 \%$ of RGTs in HPAH ( $n=58$ families) and $5 \%$ in IPAH ( $n=126$ patients), but are lower than the proportion reported by COGAN et al. [23]: 33\% in a smaller series of families $(n=12)$. Among these mutations, the deletion of BMPR2 exon 10 and exons 11-13 have been previously reported [19, 20], but the other four RGTs are novel.

We also found two splice-site mutations in this study due to mutations in introns affecting mRNA splicing. One was reported for the first time in our study, and in silico prediction was in favour of a deleterious effect, based on splice-site software predictions, but the consequences on mRNA maturation were not tested in vitro in this study. 21 missense mutations of BMPR2 found in Chinese IPAH and HPAH clusters were confined to exons 2, 3, 6, 8 to 9, 11 and 12. Exons 2 and 3 encode the extracellular ligand-binding domain, essential for signalling activity of BMPR2, which adopts a precisely folded conformation, exquisitely dependent on the formation of five disulphide bridges by 10 cysteine residues dispersed across exons 2 and 3. Mutations were found on cysteines 60, 84 and 117, affecting three out of the 10 conserved cysteine residues. Cysteine 60, 84 and 117 mutations have been reported previously $[7,16]$, but the substituted amino acids found in our study are different. Cysteine mutant constructs by transient transfection of GFP- or myc-tagged BMPR2 constructs demonstrated substantial cytosolic retention and significant protein misfolding, which is likely to be due to a profound loss of conformational integrity [24]. The kinase region encoded by exons 5-11 harbours 13 discrete PAH-related missense mutations seen among 305 probands (table 2), which constitutes the largest proportion of all the missense variations in this study. The kinase region is compartmentalised into 12 subdomains of variable importance for the processes of adenosine triphosphate binding, substrate recognition and phosphate group transfer [25]. X-ray crystallography revealed that a highly conserved 12 invariant amino acids motif lies within the $\sim 250-300$ amino acid core. We found several missense mutations involving key residues, such as Arg 491 and Cys 420, which are essential catalytic residues and their mutations cause a near complete abolition of signalling through the Smad pathway [24].

The variant c.-93A $>\mathrm{G}$ in the $5^{\prime}$-UTR was frequently observed and was further investigated because it is not present in databases (dbSNP, HapMap, 1000 genome). We genotyped 237 normal subjects, and found this variant at a frequency of $0.84 \%$ for the $G$ allele in the different Chinese populations tested in the panel, clearly showing that it is a rare variant.

Our findings suggest haploinsufficiency as the predominant molecular mechanism underlying BMPR2 predisposition to $\mathrm{HPAH}$ and IPAH, as mutations identified are likely to inactivate the receptor [17]. Additional clinical characteristics are of note in the group of patients studied. The first is that the mean age of patients was around 8 yrs younger than the age of patients with IPAH or HPAH in other published series $[15,26]$, although clinical and haemodynamic criteria for inclusion in the study were similar. It is difficult to speculate on the reasons for such a difference, since both the environmental and genetic background were different in these studies, but it shows the severity of the disease occurring in the Chinese Han population, and suggests an increased pressure of geographically and population-specific factors, in conjunction with known genetic factors when BMPR2 mutations are present, or unknown in the case of IPAH. In addition, HPAH patients are $\sim 14$ yrs younger than the IPAH patients, a difference in the same range as that found in other studies [15]. Considering that the BMPR2 mutation rate is $53.3 \%$ and $14.5 \%$ in $\mathrm{HPAH}$ and IPAH, respectively, the inherited risk may be the main factor driving earlier detection among $\mathrm{HPAH}$ patients. Another striking result is the difference in the proportion of mutation carriers between males and females, since $25.3 \%$ of males were mutation carriers and only $12.8 \%$ of females $(p=0.008)$. This proportion contrasts with results reported by GIRERD et al. [27], where the proportion of mutated patients was similar in both sexes and was $30.1 \%$, close to the proportion found in male 


\begin{tabular}{lcr} 
TABLE 6 & $\begin{array}{l}\text { Sex difference between mutation carriers and } \\
\text { noncarriers in the idiopathic and hereditable } \\
\text { pulmonary arterial hypertension cohorts }\end{array}$ \\
\hline Sex & Mutation carriers & Nonmutation carriers \\
\hline Female & $28(12.8)^{\star *}$ & $190(87.2)$ \\
Male & $22(25.3)$ & $65(74.7)$ \\
Total & $50(16.4)$ & $255(83.6)$ \\
\hline & \\
Data are presented as $n(\%) .{ }^{* *}: p<0.01$ versus male mutation carriers (Chi- \\
squared test).
\end{tabular}

Chinese patients. A differential effect on embryo survival was proposed to explain the female/male ratio of $\mathrm{PAH}$, but results in previously published series did not favour such a hypothesis as the proportion of female and male carriers was found to be similar. In our study, the significant difference in the proportion of female carriers and male carriers (table 6) would suggest two different hypotheses: 1) there is a female-specific aetiological factor for $\mathrm{PAH}$, decreasing the relative proportion of BMPR2 carriers among female PAH patients, or 2) female embryos carrying the mutation would be disadvantaged either by meiotic drive or later in embryo development, an hypothesis at discrepancy with previously proposed hypotheses.

It has been reported that the age at diagnosis is $\sim 10$ yrs earlier in $\mathrm{PAH}$ patients with mutations than in those without mutations in the BMPR2 gene [15], but in a smaller series of patients, AUsTiN et al. [26], found that the younger age at diagnosis in mutation carriers was confined to female patients. In our series of patients, we confirmed that the mean age at diagnosis is $\sim 6$ yrs younger in mutation carriers than in noncarriers, but while the relationship was significant in females, there was only a tendency present in males. Therefore, since the age difference is also present in male carriers, the lack of statistical power due to the smaller size of the male group is the most likely explanation for this discrepancy.

In conclusion, our study reports an extensive molecular investigation of the BMPR2 gene in Chinese patients with $\mathrm{PAH}$. The overall genetics of $\mathrm{PAH}$ in Chinese patients was similar to that of other populations already explored for this disease, although a few characteristics were noteworthy: we found polymorphisms of the BMPR2 gene that are important to know in the context of genetic investigation of the disease in Chinese patients. The predisposing effect of the BMPR2 gene was similar to that of other populations, and the younger age at diagnosis suggests that the BMPR2 mutation constitutes the first hit that increases the probability of an early onset of the disease. This observation is similar to hereditary predisposition to cancer, exemplified by the two-hit hypothesis proposed by KNUDSON [28] for tumour suppressor genes, which corresponds to a germ-line loss of function for one allele and a somatic loss of the second allele. In the case of PAH, the second event might be, as well as genetic, either environmental as suggested by somatic chromosomal abnormalities found in the lungs of PAH subjects [29], or epigenetic, potentially leading to decreased expression of the normal BMPR2 allele [30].

\section{SUPPORT STATEMENT}

This study was supported by the Shen Kang Major Joint Research Project On Emerging Technology In Municipal Hospital (shdc12010102) and Non-governmental International Cooperation Project (08410701600) of the Shanghai Science and Technology Division.

\section{STATEMENT OF INTEREST}

A statement of interest for Z-C. Jing can be found at www.erj. ersjournals.com/site/misc/statements.xhtml

\section{REFERENCES}

1 Rich S, Dantzker DR, Ayres SM, et al. Primary pulmonary hypertension. A national prospective study. Ann Intern Med 1987; 107: 216-223.

2 Sztrymf B, Yaïci A, Girerd B, et al. Genes and pulmonary arterial hypertension. Respiration 2007; 74: 123-132.

3 Humbert M, Sitbon O, Chaouat A, et al. Pulmonary arterial hypertension in France: results from a national registry. Am J Respir Crit Care Med 2006; 173: 1023-1030.

4 Loyd JE, Butler MG, Foroud TM, et al. Genetic anticipation and abnormal gender ratio at birth in familial primary pulmonary hypertension. Am J Respir Crit Care Med 1995; 152: 93-97.

5 Deng Z, Morse JH, Slager SL, et al. Familial primary pulmonary hypertension (gene PPH1) is caused by mutations in the bone morphogenetic protein receptor-II gene. Am J Hum Genet 2000; 67: 737-744.

6 Lane KB, Machado RD, Pauciulo MW, et al. Heterozygous germline mutations in BMPR2, encoding a TGF- $\beta$ receptor, cause familial primary pulmonary hypertension. Nat Genet 2000; 26: 81-84.

7 Thomson JR, Machado RD, Pauciulo MW, et al. Sporadic primary pulmonary hypertension is associated with germline mutations of the gene encoding BMPR-II, a receptor member of the TGF- $\beta$ family. J Med Genet 2000; 37: 741-745.

8 Morisaki H, Nakanishi N, Kyotani S, et al. BMPR2 mutations found in Japanese patients with familial and sporadic primary pulmonary hypertension. Hum Mutat 2004; 23: 632.

9 Simonneau G, Robbins IM, Beghetti M, et al. Updated clinical classification of pulmonary hypertension. J Am Coll Cardiol 2009; 54: S43-S54.

10 Massagué J, Chen YG. Controlling TGF- $\beta$ signaling. Genes Dev 2000; 14: 627-644.

11 Adachi-Yamada $\mathrm{T}$, Nakamura $\mathrm{M}$, Irie $\mathrm{K}$, et al. p38 mitogenactivated protein kinase can be involved in transforming growth factor $\beta$ superfamily signal transduction in Drosophila wing morphogenesis. Mol Cell Biol 1999; 19: 2322-2329.

12 Simonneau G, Galiè N, Rubin LJ, et al. Clinical classification of pulmonary hypertension. J Am Coll Cardiol 2004; 43: 5S-12S.

13 Human Gene Variation Society. Nomenclature for the description of sequence variants. www.hgvs.org/mutnomen Date last updated: August, 2011

14 Machado RD, Aldred MA, James V, et al. Mutations of the TGF- $\beta$ type II receptor BMPR2 in pulmonary arterial hypertension. Hum Mutat 2006; 27: 121-132.

15 Sztrymf B, Coulet F, Girerd B, et al. Clinical outcomes of pulmonary arterial hypertension in carriers of BMPR2 mutation. Am J Respir Crit Care Med 2008; 177: 1377-1383.

16 Machado RD, Eickelberg O, Elliott CG, et al. Genetics and genomics of pulmonary arterial hypertension. J Am Coll Cardiol 2009; 54: S32-S42.

17 Machado RD, Pauciulo MW, Thomson JR, et al. BMPR2 haploinsufficiency as the inherited molecular mechanism for primary pulmonary hypertension. Am J Hum Genet 2001; 68: 92-102.

18 Wang $\mathrm{H}$, Cui $\mathrm{QQ}$, Sun $\mathrm{K}$, et al. Identities and frequencies of BMPR2 mutations in Chinese patients with idiopathic pulmonary arterial hypertension. Clin Genet 2010; 77: 189-192. 
19 Aldred MA, Vijayakrishnan J, James V, et al. BMPR2 gene rearrangements account for a significant proportion of mutations in familial and idiopathic pulmonary arterial hypertension. Hum Mutat 2006; 27: 212-213.

20 Trembath RC, Thomson JR, Machado RD, et al. Clinical and molecular genetic features of pulmonary hypertension in patients with hereditary hemorrhagic telangiectasia. N Engl J Med 2001; 345: 325-334.

21 Sunyaev S, Ramensky V, Koch I, et al. Prediction of deleterious human alleles. Hum Mol Genet 2001; 10: 591-597.

22 Cogan JD, Vnencak-Jones CL, Phillips JR, et al. Gross BMPR2 gene rearrangements constitute a new cause for primary pulmonary hypertension. Genet Med 2005; 7: 169-174.

23 Cogan JD, Pauciulo MW, Batchman AP, et al. High frequency of $B M P R 2$ exonic deletions/duplications in familial pulmonary arterial hypertension. Am J Respir Crit Care Med 2006; 174: 590-598.

24 Rudarakanchana N, Flanagan JA, Chen $\mathrm{H}$, et al. Functional analysis of bone morphogenetic protein type II receptor mutations underlying primary pulmonary hypertension. Hum Mol Genet 2002; 11: 1517-1525.

25 Hanks SK, Hunter T. Protein kinases 6. The eukaryotic protein kinase superfamily: kinase (catalytic) domain structure and classification. FASEB J 1995; 9: 576-596.

26 Austin ED, Phillips JA, Cogan JD, et al. Truncating and missense BMPR2 mutations differentially affect the severity of heritable pulmonary arterial hypertension. Respir Res 2009; 28: 87.

27 Girerd B, Montani D, Eyries M, et al. Absence of influence of gender and $B M P R 2$ mutation type on clinical phenotypes of pulmonary arterial hypertension. Respir Res 2010; 10: 73.

28 Knudson AG Jr. Mutation and cancer: statistical study of retinoblastoma. Proc Natl Acad Sci USA 1971; 68: 820-823.

29 Aldred MA, Comhair SA, Varella-Garcia M, et al. Somatic chromosome abnormalities in the lungs of patients with pulmonary arterial hypertension. Am J Respir Crit Care Med 2010; 182: 1153-1160.

30 Hamid R, Cogan JD, Hedges LK, et al. Penetrance of pulmonary arterial hypertension is modulated by the expression of normal BMPR2 allele. Hum Mutat 2009; 30: 649-654. 